\title{
Design and Control of Novel Tri-rotor UAV
}

\author{
Mohamed Kara Mohamed \\ School of Electrical and \\ Electronic Engineering \\ The University of Manchester \\ Manchester, UK, M13 9PL \\ Email: Mohamed.KaraMohamed@postgrad.manchester.ac.uk
}

\author{
Alexander Lanzon \\ School of Electrical and \\ Electronic Engineering \\ The University of Manchester \\ Manchester, UK, M13 9PL \\ Email: Alexander.Lanzon@manchester.ac.uk
}

\begin{abstract}
Tri-rotor UAVs are more efficient compared to quadrotors in regard to their size and power requirement, yet, they are more challenging in terms of control and stability. In this paper, we propose the design and control of a novel tri-rotor UAV. The proposed platform is designed to achieve six degree of freedom using a thrust vectoring technique with the highest level of flexibility, manoeuvrability and minimum requirement of power. The proposed tri-rotor has a triangular shape of three arms where at the end of each arm, a fixed pitch propeller is driven by a DC motor. A tilting mechanism is employed to tilt the motor-propeller assembly and produce thrust in the desired direction. The three propellers can be tilted independently to achieve full authority of torque and force vectoring. A feedback linearization associated with $\mathscr{H}_{\infty}$ loop shaping design is used to synthesize a controller for the system. The results are verified via simulation.
\end{abstract}

\section{BACKGROUND AND MOTIVATION}

In recent decades, Unmanned Aerial Vehicles (UAVs) have attracted growing attention in research due to their wide applications and large potential [1], [2]. Aiming for more efficiency in term of size, autonomy, payload capacity and maneuverability among other factors, various conventional and non-conventional structure designs and configurations of UAV systems are proposed [3], [4] and the literature therein. One such design that attracts increasing interest is the vertical-takeoff-and-landing (VTOL) tri-rotor configuration.

Tri-rotor vehicles are systems with three rotors arrangement. This configuration has been proposed as less-expensive with more flexibility and great agility [4], [5]. Compared to quadrotors, tri-rotor UAVs are smaller in size, less complex, less costly and have longer flight time due to the reduction in number of motors [6], which makes tri-rotor vehicles ideal for deployment in various research projects and missions [7].

Thrust vectoring has been used in designs to maximize the capability of UAVs [8]. Thrust vectoring is of significant benefit in some applications to arbitrarily orient the vehicle body with respect to the vehicle acceleration vector, e.g., for aircrafts carrying directional sensors that have to be pointed at targets in the earth reference frame [9]. In addition, thrust vectoring mechanism is used to give UAVs the capability of taking-off and landing in very narrow areas [10]. In small aircrafts and UAVs, a simple technique of tilt-rotor mechanism can be used to obtain thrust vectoring, where propulsion units are inclined in certain angles using an additional control motor to get the desired thrust in different directions.
In tri-rotor systems, tilt-rotor mechanism is used to control the horizontal forces and yaw torque of the vehicle. Attitude control of these vehicles is more challenging compared to quadrotor systems due to gyroscopic and coriolis terms. Typically, one rotor only, referred to as the tail rotor, has the ability to tilt to control the yaw moment of the vehicle, see for example [7], [5]. In [11], the attitude of tri-rotor UAVs is controlled by using differential thrust concept. In [4], all rotors of the proposed tri-rotor system are tilting simultaneously by the same angle to attain yaw control. The UAV in [12] controls the yaw angle by differentially tilting the two main rotors in the plane of symmetry. In this reference, a fixed up-right propeller is used at the tail to control the pitch moment.

Few researchers have identified the structure of tri-rotor UAV combined with full independent tilt-rotor capability. In this paper, we propose a novel tri-rotor platform, herein referred to as the Tri-rotor UAV, and then we discuss the design and control of the proposed system. The proposed vehicle can achieve full authority of torque and force vectoring by employing three rotors and three servos for tilt-rotor mechanism. This structure gives the vehicle high level of maneuverability and flexibility for translational motion as well as attitude control.

The rest of the paper is organized as follows. In Section II, a functional description of the vehicle and its design is discussed. A mathematical model that captures the dynamics of the UAV and govern the behaviour of the system is derived in Section III. The control system design is presented in Section IV and the simulation results is shown in Section V. The paper ends by conclusion in Section VI.

\section{System Structure And Design}

The structure of the proposed Tri-rotor UAV is depicted in Figure 1. The vehicle has a triangular structure of three arms and at the end of each arm, a force generating unit is mounted to produce part of the required controlling force/torque. All three arms are identical of length $l$ and the three force generating units are also identical. Each force generating unit consists of a fixed pitch propeller driven by a Brushless DC (BLDC) motor to generate thrust. The three motors can be powered by a single battery pack or three separate packs located at the center of the body. The propeller-motor assembly is attached to the body arm via a servo motor that can rotate in a vertical plane to tilt the propeller-motor assembly with an angle $\alpha_{s_{i}}$ in 


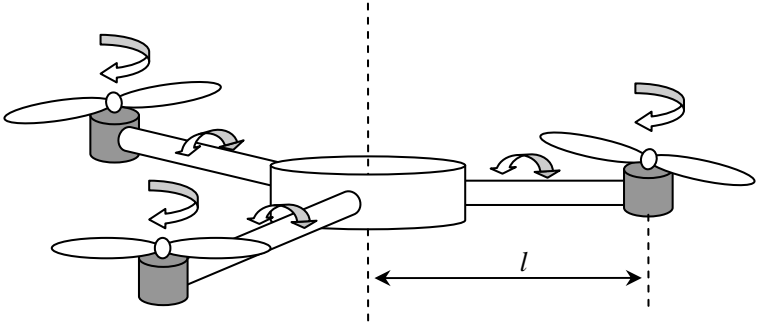

Figure 1. The design of the Tri-rotor UAV (3D view).

the range $\frac{-\pi}{2} \leq \alpha_{s_{i}} \leq \frac{\pi}{2}, i=1,2,3$ to produce a horizontal component of the generated force, see Figure 2. All three propellers can be tilted independently to give full authority of thrust vectoring. The system has six degree of freedom in which all movements can be achieved independently and directly by changing the norm of the generated thrust and the tilting angles. This configuration enables the vehicle body to stay aligned in the required direction regardless of the movement the UAV makes.

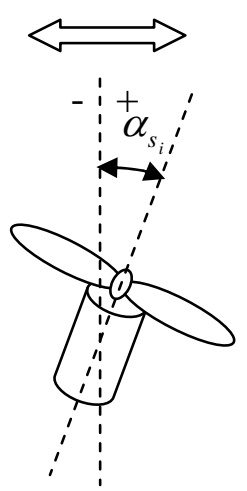

Figure 2. Front view of one arm.

\section{Mathematical Modeling}

To develop the dynamic model of the UAV, we consider the following right hand coordinate systems shown in Figure 3: $e$ : the generalized earth coordinate system of axes $X_{e}, Y_{e}, Z_{e}$. $b$ : the body fixed coordinate system in which the origin coincides with the centre of mass of the UAV. The axes of frame $b$ are denoted by $X_{b}, Y_{b}, Z_{b}$. In addition, we choose three right hand coordinate systems $l_{i}$ of axes $X_{l_{i}}, Y_{l_{i}}, Z_{l_{i}}$ with $i=1,2,3$. These coordinate systems are termed as local coordinate systems and located at the locations of the three propellers, see Figure 4. The origin of each local coordinate system coincides with the joining point between the UAV arm and the propulsion unit where $X_{l_{i}}$ is extended outside the $i^{\text {th }}$ arm of the UAV and $Z_{l_{i}}$ is along the BLDC motor shaft axis when the tilting angle is zero.

The rotation matrices between the defined coordinate systems are denoted by:

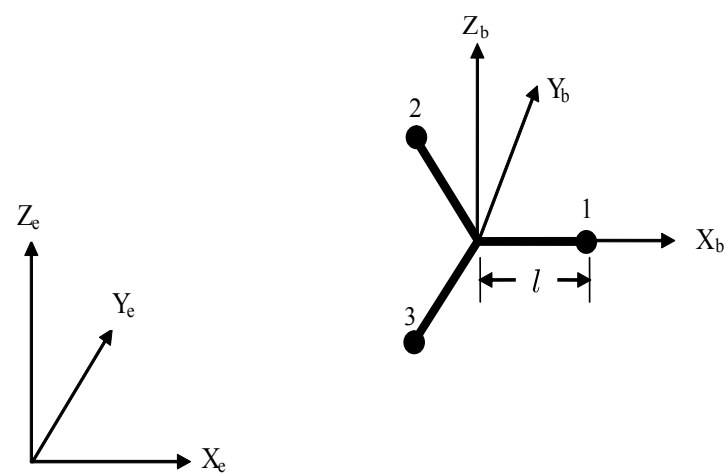

Figure 3. Coordinate systems used to develop the UAV dynamic model.

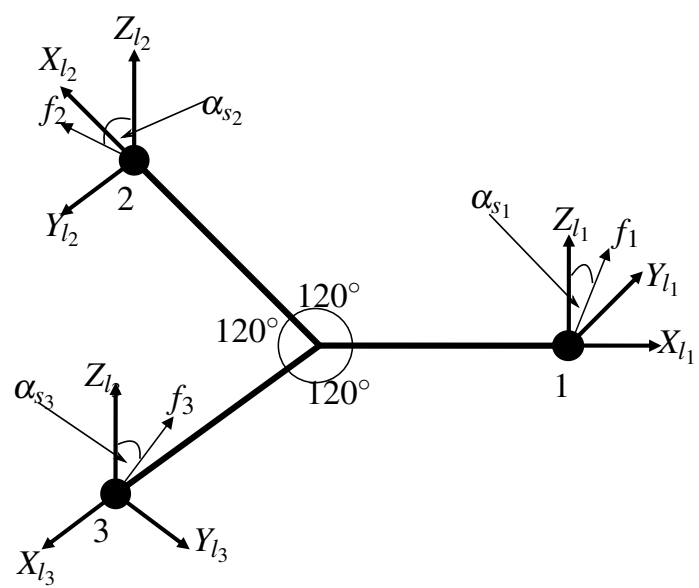

Figure 4. Local coordinate systems at the three propulsion units.

$\boldsymbol{R}_{e}^{b}$ : the rotational matrix from frame $e$ to frame $b$.

$\boldsymbol{R}_{l_{i}}^{b}$ : the rotational matrix from coordinate system $l_{i}$ to coordinate system $b, i=1,2,3$.

In the sequel, we use superscript $b, e$ and $l_{i}$ to denote the coordinate system in which vectors are expressed. The subscript $i$ refers to the $i^{\text {th }}$ BLDC motor, servo motor or propeller as applies where $i=1,2,3$.

In order to obtain the dynamic equations of the UAV, we need to obtain forces and torques acting on the vehicle. We assume very fast actuators and therefore the dynamics of the actuators are neglected.

\section{Forces}

There are two main forces acting on the UAV which are the propulsive force and the gravitational force.

The propulsive force: The total propulsive force $F_{p_{\Sigma}}$ is equal to the algebraic sum of the three individual propulsive forces generated from propellers. The individual propulsive forces of the three propellers expressed in the local coordinate systems can be written as:

$$
F_{p_{i}}^{l_{i}}=\left[\begin{array}{c}
0 \\
k_{f} \omega_{m_{i}}^{2} \sin \left(\alpha_{s_{i}}\right) \\
k_{f} \omega_{m_{i}}^{2} \cos \left(\alpha_{s_{i}}\right)
\end{array}\right], i=1,2,3 .
$$


where $k_{f}$ is the thrust to speed constant of the propeller and it is identical for all three propellers, $\omega_{m_{i}}$ is the rotational speed of the $i^{t h}$ BLDC motor (the rotational speed of the motor equals the rotational speed of the propeller) and $\alpha_{s_{i}}$ is the tilting angle of the $i^{\text {th }}$ Servo motor.

In the body coordinate system, the individual propulsive forces are given by:

$$
F_{p_{i}}^{b}=\boldsymbol{R}_{l_{i}}^{b} F_{P_{i}}^{l_{i}}, i=1,2,3 .
$$

From Figure 4, we can obtain the rotation matrices from the local coordinate systems $l_{1}, l_{2}$ and $l_{3}$ to the body coordinate system $b$ as:

$$
\begin{aligned}
\boldsymbol{R}_{l_{1}}^{b} & =\left[\begin{array}{lll}
1 & 0 & 0 \\
0 & 1 & 0 \\
0 & 0 & 1
\end{array}\right], \\
\boldsymbol{R}_{l_{2}}^{b} & =\left[\begin{array}{ccc}
-\frac{1}{2} & -\frac{\sqrt{3}}{2} & 0 \\
\frac{\sqrt{3}}{2} & -\frac{1}{2} & 0 \\
0 & 0 & 1
\end{array}\right], \\
\boldsymbol{R}_{l_{3}}^{b} & =\left[\begin{array}{ccc}
-\frac{1}{2} & \frac{\sqrt{3}}{2} & 0 \\
\frac{-\sqrt{3}}{2} & -\frac{1}{2} & 0 \\
0 & 0 & 1
\end{array}\right] .
\end{aligned}
$$

Using Equations (3) - (5), the total propulsive force is:

$$
\begin{aligned}
F_{p_{\Sigma}}^{b} & =F_{p_{1}}^{b}+F_{p_{2}}^{b}+F_{p_{3}}^{b} \\
& =k_{f} \boldsymbol{H}_{f} \rho .
\end{aligned}
$$

where

$$
\boldsymbol{H}_{f}=\left[\begin{array}{cccccc}
0 & -\frac{\sqrt{3}}{2} & \frac{\sqrt{3}}{2} & 0 & 0 & 0 \\
1 & -\frac{1}{2} & -\frac{1}{2} & 0 & 0 & 0 \\
0 & 0 & 0 & 1 & 1 & 1
\end{array}\right],
$$

and

$$
\rho=\left[\begin{array}{l}
\omega_{m_{1}}^{2} \sin \left(\alpha_{s_{1}}\right) \\
\omega_{m_{2}}^{2} \sin \left(\alpha_{s_{2}}\right) \\
\omega_{m_{3}}^{2} \sin \left(\alpha_{s_{3}}\right) \\
\omega_{m_{1}}^{2} \cos \left(\alpha_{s_{1}}\right) \\
\omega_{m_{2}}^{2} \cos \left(\alpha_{s_{2}}\right) \\
\omega_{m_{3}}^{2} \cos \left(\alpha_{s_{3}}\right)
\end{array}\right] .
$$

The gravity force: The gravitational force in the generalized earth coordinate system is given as:

$$
F_{g}^{e}=\left[\begin{array}{c}
0 \\
0 \\
-g M_{t o t}
\end{array}\right]
$$

where $g$ is the gravitational acceleration and $M_{t o t}$ is the total mass of the UAV.

In the body coordinate system, we have:

$$
F_{g}^{b}=\boldsymbol{R}_{e}^{b} F_{g}^{e} .
$$

Using the general notation of rotation angles for the UAV attitude: Roll $\phi_{v}$, Pitch $\theta_{v}$ and Yaw $\psi_{v}$ around the axes $X_{e}, Y_{e}$ and $Z_{e}$ respectively, the gravity force in the body system is given by:

$$
F_{g}^{b}=g M_{t o t} H_{g}
$$

where

$$
H_{g}=\left[\begin{array}{c}
\sin \left(\theta_{v}\right) \\
-\sin \left(\phi_{v}\right) \cos \left(\theta_{v}\right) \\
-\cos \left(\phi_{v}\right) \cos \left(\theta_{v}\right)
\end{array}\right] .
$$

Now, the total force acting on the UAV and expressed in the body coordinate system is:

$$
\begin{aligned}
F^{b} & =F_{p_{\Sigma}}^{b}+F_{g}^{b} \\
& =k_{f} \boldsymbol{H}_{f} \rho+g M_{t o t} H_{g} .
\end{aligned}
$$

\section{Torques}

The two main torques acting on the UAV are the propulsive torque and the drag torque.

The propulsive torque: The propulsive torque is the torque resulting from the generated propulsive force around the center of mass of the vehicle. For the case of the Tri-rotor $\mathrm{UAV}$, we have three identical arms and then the components of the propulsive torque are:

$$
\tau_{p_{i}}^{b}=\bar{l}_{i}^{b} \times F_{p_{i}}^{b}, i=1,2,3 .
$$

where $\bar{l}_{i}^{b}$ is the vector of the $i^{t h}$ arm between the center of mass of the UAV and the propulsion unit expressed in the body coordinate system. $F_{b_{i}}^{b}$ is obtained from Eq. (2).

Now, the total propulsive torque expressed in the body coordinate system is:

$$
\begin{aligned}
\tau_{p_{\Sigma}}^{b} & =\tau_{p_{1}}^{b}+\tau_{p_{2}}^{b}+\tau_{p_{3}}^{b} \\
& =k_{f} \boldsymbol{H}_{t} \rho
\end{aligned}
$$

where

$$
\boldsymbol{H}_{t}=l\left[\begin{array}{cccccc}
0 & 0 & 0 & 0 & \frac{\sqrt{3}}{2} & -\frac{\sqrt{3}}{2} \\
0 & 0 & 0 & -1 & \frac{1}{2} & \frac{1}{2} \\
1 & 1 & 1 & 0 & 0 & 0
\end{array}\right],
$$

$l$ is the length of the vehicle's arm vector measured between the center of mass of the UAV and the propulsion unit (identical for the three arms) and $\rho$ is defined in Eq. (9).

The drag torque: The drag torque is defined as the torque resulting from the aerodynamic drag forces exerted by the ambient fluid (air) on the propeller. Drag torque is in the opposite direction to the direction of rotation. In our case, the resulting drag torque on the $i^{\text {th }}$ propeller can be approximated by $\tau_{d_{i}}=-k_{t} \omega_{m_{i}}^{2}$ where we consider the BLDC motors drives the propeller directly and $k_{t}$ is the drag torque to speed constant resulting from the rotation of the propeller. In the local coordinate systems $l_{i}$, the drag torque can be written as:

$$
\tau_{d_{i}}^{l_{i}}=\left[\begin{array}{c}
0 \\
-k_{t} \omega_{m_{i}}^{2} \sin \left(\alpha_{s_{i}}\right) \\
-k_{t} \omega_{m_{i}}^{2} \cos \left(\alpha_{s_{i}}\right)
\end{array}\right], i=1,2,3 .
$$

In the body coordinate system, the individual drag torques can be represented as:

$$
\tau_{d_{i}}^{b}=\boldsymbol{R}_{l_{i}}^{b} \tau_{d_{i}}^{l_{i}}
$$


Using definitions (3) - (5), the total drag torque in the body system is given by:

$$
\begin{aligned}
\tau_{d_{\Sigma}}^{b} & =\tau_{d_{1}}^{b}+\tau_{d_{2}}^{b}+\tau_{d_{3}}^{b} \\
& =-k_{t} \boldsymbol{H}_{f} \boldsymbol{\rho},
\end{aligned}
$$

where $\boldsymbol{H}_{f}$ and $\rho$ are defined in (8) and (9) respectively.

Now, the total torque acting on the Tri-rotor and expressed in the body coordinate system is:

$$
\begin{aligned}
\tau^{b} & =\tau_{p_{\Sigma}}^{b}+\tau_{d_{\Sigma}}^{b} \\
& =\left(k_{f} \boldsymbol{H}_{t}-k_{t} \boldsymbol{H}_{f}\right) \rho .
\end{aligned}
$$

Dynamic Model: Assuming that the Tri-rotor UAV is a rigid body of fixed mass, the vehicle's motion can be described by the Newton-Euler second's law in the body coordinate system as:

for translational motion:

$$
F^{b}=M_{t o t}\left(\dot{v}_{v}^{b}+\boldsymbol{S}\left(\omega_{v}^{b}\right) v_{v}^{b}\right)
$$

for rotational motion:

$$
\tau^{b}=\boldsymbol{I}_{v}^{b} \dot{\boldsymbol{\omega}}_{v}^{b}+\boldsymbol{S}\left(\boldsymbol{\omega}_{v}^{b}\right) \boldsymbol{I}_{v}^{b} \boldsymbol{\omega}_{v}^{b}
$$

where $v_{v}^{b}$ is translational velocity of the UAV, $\omega_{v}^{b}$ is the angular velocity of the UAV, $\boldsymbol{S}\left(\boldsymbol{\omega}^{b}\right)$ is the skew matrix of the vector $\omega_{v}^{b}$ and $\boldsymbol{I}_{v}^{b}$ is the inertia matrix of the UAV all with respect to the fixed body coordinate system. Assuming no mass change over time, $\boldsymbol{I}_{v}^{b}$ is fixed.

Now, Substituting $F^{b}$ and $\tau^{b}$ from (15) and (25) gives:

$$
\begin{array}{r}
k_{f} \boldsymbol{H}_{f} \rho+g M_{t o t} H_{g}=M_{t o t}\left(\dot{v}_{v}^{b}+\boldsymbol{S}\left(\omega_{v}^{b}\right) v_{v}^{b}\right) \\
\left(k_{f} \boldsymbol{H}_{t}-k_{t} \boldsymbol{H}_{f}\right) \rho=\boldsymbol{I}_{v}^{b} \dot{\omega}_{v}^{b}+\boldsymbol{S}\left(\omega_{v}^{b}\right) \boldsymbol{I}_{v}^{b} \omega_{v}^{b}
\end{array}
$$

Let $\eta_{v}$ and $\lambda_{v}^{e}$ be the attitude vector and the position vector of the UAV related to the earth coordinate system and defined as:

$$
\eta_{v}=\left[\begin{array}{c}
\phi_{v} \\
\theta_{v} \\
\psi_{v}
\end{array}\right], \lambda_{v}^{e}=\left[\begin{array}{l}
x_{v} \\
y_{v} \\
z_{v}
\end{array}\right] .
$$

To fully describe the dynamic equations of the UAV, we have the following relations from [13]:

$$
\begin{aligned}
& \dot{\eta}_{v}=\boldsymbol{\Psi} \omega_{v}^{b} \\
& \dot{\lambda}_{v}^{e}=\left(\boldsymbol{R}_{e}^{b}\right)^{-1} v_{v}^{b}
\end{aligned}
$$

where $\boldsymbol{\Psi}$ is the rotational matrix between the angular velocity expressed in the body coordinate system $\omega_{v}^{b}$ and the angular velocity in the earth coordinate system $\dot{\eta}_{v} . \Psi$ is given in [13] as:

$$
\Psi=\left[\begin{array}{ccc}
1 & \sin \left(\phi_{v}\right) \tan \left(\theta_{v}\right) & \cos \left(\phi_{v}\right) \tan \left(\theta_{v}\right) \\
0 & \cos \left(\phi_{v}\right) & -\sin \left(\phi_{v}\right) \\
0 & \sin \left(\phi_{v}\right) \sec \left(\theta_{v}\right) & \cos \left(\phi_{v}\right) \sec \left(\theta_{v}\right)
\end{array}\right], \frac{-\pi}{2}<\theta_{v}<\frac{\pi}{2} .
$$

From the properties of the rotation matrix we have:

$$
\left(\boldsymbol{R}_{e}^{b}\right)^{-1}=\boldsymbol{R}_{b}^{e} .
$$

where $\boldsymbol{R}_{b}^{e}$ is the rotation matrix from the body coordinate system $b$ to the earth coordinate system $e$.
Finally, from Equations (28) - (34), the dynamic model of the UAV can be written as:

$$
\begin{aligned}
& \dot{v}^{b}{ }_{v}=g H_{g}-\boldsymbol{S}\left(\boldsymbol{\omega}_{v}^{b}\right) v_{v}^{b}+\frac{k_{f}}{M_{t o t}} \boldsymbol{H}_{f} \boldsymbol{\rho} \\
& \dot{\omega}^{b}{ }_{v}=-\left(\boldsymbol{I}_{v}^{b}\right)^{-1} \boldsymbol{S}\left(\boldsymbol{\omega}_{v}^{b}\right) \boldsymbol{I}_{v}^{b} \omega_{v}^{b}+\left(\boldsymbol{I}_{v}^{b}\right)^{-1}\left(k_{f} \boldsymbol{H}_{t}-k_{t} \boldsymbol{H}_{f}\right) \rho \\
& \dot{\eta}_{v}=\boldsymbol{\Psi} \boldsymbol{\omega}_{v}^{b} \\
& \dot{\lambda}_{v}^{e}=\boldsymbol{R}_{b}^{e} v_{v}^{b}
\end{aligned}
$$

This model of the UAV is written in the compact form in which every state variable is a vector of three components, i.e., $x \in \mathbb{R}^{12}$, where:

$$
v_{v}^{b}=\left[\begin{array}{c}
u \\
v \\
w
\end{array}\right], \omega_{v}^{b}=\left[\begin{array}{l}
p \\
q \\
r
\end{array}\right], \eta_{v}=\left[\begin{array}{l}
\phi_{v} \\
\theta_{v} \\
\psi_{v}
\end{array}\right], \lambda_{v}^{e}=\left[\begin{array}{c}
x_{v} \\
y_{v} \\
z_{v}
\end{array}\right] .
$$

Equations (35) - (38) show a nonlinear model with coupling between the translational and rotational dynamics of the UAV. Moreover, there is coupling between inputs and output channels in which all inputs act on all outputs. The system coupling along with the nonlinearity of the system makes the control design of the proposed Tri-rotor UAV a real challenge compared with other UAV configurations. On the other hand, if we consider the control problem of the UAV to be position tracking with attitude regulating, then the system is square in which we have six actuators (three BLDC motor speeds and three servo angles) and six outputs (3D position and three attitude angles). This highlights the positive aspect of the proposed configuration in terms of controller design compared to other UAV systems that are in general underactuated systems such as quadrotors.

\section{Control System Design}

In this section, we synthesize a controller for the Tri-rotor UAV. The system is linearized using input-output feedback method and then an $\mathscr{H}_{\infty}$ controller is designed for the linearized plant. For simplicity of expression, the superscript $b$ and $e$ as well as the subscript $v$ are not written unless it is necessary to avoid ambiguity.

We consider the vector $\rho$ as the input vector for the UAV system, i.e., $u=\rho$, and we choose the output as:

$$
y=\left[\begin{array}{l}
\eta \\
\lambda
\end{array}\right]
$$

To implement input-output feedback linearization, we have:

$$
\dot{y}=y^{(1)}=\left[\begin{array}{c}
\dot{\eta} \\
\dot{\lambda}
\end{array}\right]=\left[\begin{array}{c}
\boldsymbol{\Psi} \omega \\
\boldsymbol{R}_{b}^{e} v_{v}
\end{array}\right]
$$

and

$$
\ddot{y}=y^{(2)}=\left[\begin{array}{c}
\dot{\boldsymbol{\Psi}} \omega+\boldsymbol{\Psi} \dot{\boldsymbol{\omega}} \\
\left(\dot{\boldsymbol{R}}_{b}^{e}\right) v+\boldsymbol{R}_{b}^{e} \dot{v}
\end{array}\right]
$$

From the general properties of the rotation matrix, we have:

$$
\dot{\boldsymbol{R}}_{b}^{e}=\boldsymbol{R}_{b}^{e} \boldsymbol{S}\left(\omega^{b}\right)
$$


and then we write:

$$
\begin{aligned}
& y^{(2)}=\left[\begin{array}{c}
\dot{\boldsymbol{\Psi}} \omega+\boldsymbol{\Psi}\left(-\boldsymbol{I}^{-1} \boldsymbol{S}(\omega) \boldsymbol{I} \omega+\boldsymbol{I}^{-1}\left(k_{f} H_{t}-k_{t} \boldsymbol{H}_{f}\right) \rho\right) \\
\boldsymbol{R}_{b}^{e} \boldsymbol{S}(\omega) v+\boldsymbol{R}_{b}^{e}\left(g H_{g}-\boldsymbol{S}(\omega) v+\frac{k_{f}}{M_{t o t}} \boldsymbol{H}_{f} \rho\right)
\end{array}\right] \\
& =\left[\begin{array}{c}
\left(\dot{\boldsymbol{\Psi}}-\boldsymbol{\Psi} \boldsymbol{I}^{-1} \boldsymbol{S}(\boldsymbol{\omega}) \boldsymbol{I}\right) \boldsymbol{\omega} \\
g \boldsymbol{R}_{b}^{e} H_{g}
\end{array}\right]+\left[\begin{array}{c}
\boldsymbol{\Psi} \boldsymbol{I}^{-1}\left(k_{f} \boldsymbol{H}_{t}-k_{t} \boldsymbol{H}_{f}\right) \\
\frac{k_{f}}{M_{t o t}} \boldsymbol{R}_{b}^{e} \boldsymbol{H}_{f}
\end{array}\right] \rho
\end{aligned}
$$

where

$$
\dot{\Psi}=\frac{\partial \Psi}{\partial \phi_{v}} \dot{\phi}_{v}+\frac{\partial \Psi}{\partial \theta_{v}} \dot{\theta}_{v}
$$

and $\dot{\phi}_{v}, \dot{\theta}_{v}$ are obtained from Eq. (37) as:

$$
\dot{\eta}=\left[\begin{array}{c}
\dot{\phi}_{v} \\
\dot{\theta}_{v} \\
\dot{\psi}_{v}
\end{array}\right]=\Psi \omega^{b}
$$

We define the matrix $\boldsymbol{\beta}(x)$ as:

$$
\boldsymbol{\beta}(x)=\left[\begin{array}{c}
\boldsymbol{\Psi} \boldsymbol{I}^{-1}\left(k_{f} H_{t}-k_{t} \boldsymbol{H}_{f}\right) \\
\frac{k_{f}}{M_{t o t}} \boldsymbol{R}_{b}^{e} \boldsymbol{H}_{f}
\end{array}\right]
$$

We have $\operatorname{det}[\boldsymbol{\beta}(x)] \neq 0$ and the inverse $\boldsymbol{\beta}^{-1}(x)$ exists always ${ }^{1}$ for all $x \in \mathbb{R}^{12}$ where $x$ represents the states of the system in the compact form:

$$
x=\left[\begin{array}{llll}
v & \omega & \eta & \lambda
\end{array}\right]^{T}
$$

The relative degree of the system in the compact form is $r=$ $r_{1}+r_{2}=2+2=4$ which is equal to the number of states in the compact form of the dynamic equations, and there is no zero dynamics. Choosing any other set of outputs will generate zero dynamics and this justifies the selection of the output set $y=\left[\begin{array}{ll}\eta & \lambda\end{array}\right]^{T}$. To linearize the system, we choose a new control input $\vartheta=\left[\begin{array}{l}\vartheta_{1} \\ \vartheta_{2}\end{array}\right]$, and we write our desired linearized dynamics as:

$$
y^{(2)}=\vartheta
$$

From Eq. (43) we can write the feedback linearisation law as:

$$
u=\boldsymbol{\beta}^{-1}\left(\vartheta-\left[\begin{array}{c}
\left(\dot{\boldsymbol{\Psi}}-\boldsymbol{\Psi} \boldsymbol{I}^{-1} \boldsymbol{S}(\boldsymbol{\omega}) \boldsymbol{I}\right) \omega \\
g \boldsymbol{R}_{b}^{e} H_{g}
\end{array}\right]\right) .
$$

The centralized input-output feedback linearization handles the coupling without the need for strict assumption on operating point to decouple the system. The linearized model in the compact form is given as:

$$
\begin{aligned}
& \dot{\zeta}=\left[\begin{array}{llll}
0 & 1 & 0 & 0 \\
0 & 0 & 0 & 0 \\
0 & 0 & 0 & 1 \\
0 & 0 & 0 & 0
\end{array}\right] \zeta+\left[\begin{array}{ll}
0 & 0 \\
1 & 0 \\
0 & 0 \\
0 & 1
\end{array}\right] \vartheta \\
& y=\left[\begin{array}{llll}
1 & 0 & 0 & 0 \\
0 & 0 & 1 & 0
\end{array}\right] \zeta
\end{aligned}
$$

\footnotetext{
${ }^{1}$ It is always assumed that $-\pi / 2 \leq \theta_{v} \leq \pi / 2$.
}

where

$$
\zeta=\left[\begin{array}{c}
\eta \\
\dot{\eta} \\
\lambda \\
\dot{\lambda}
\end{array}\right] \in \mathbb{R}^{12}, y=\left[\begin{array}{l}
\eta \\
\lambda
\end{array}\right] \in \mathbb{R}^{6}, \vartheta=\left[\begin{array}{l}
\vartheta_{1} \\
\vartheta_{2}
\end{array}\right] \in \mathbb{R}^{6}
$$

The linearized plant is a double integrator representing single degree of freedom for transitional and rotational motion. The $\mathscr{H}_{\infty}$ loop-shaping design is invoked to synthesize a controller for the linearized system. For selection of weights to shape the linearized plant, we consider the following control design specifications:

- high loop-gain at low frequency for good reference tracking and disturbance rejection.

- low loop-gain at high frequency for robustness against unmodeled dynamics and output measurement noise.

- reasonable bandwidth for fast response.

An algorithm proposed in [14] is invoked to simultaneously optimize the synthesis of loop-shaping weights and a stabilizing controller. This algorithm captures the design specification listed above in a systematic manner while trying to maximize the robust stability margin of the closed-loop system. We fix the post-compensator weight to a low-pass filter on all channels and use the algorithm to optimize the pre-compensator weights for all channels. The optimized precompensator for each channel is $w_{2}=40 /(s+25)$, where $W_{2}=w_{2} I_{6}$. The achieved robust stability margin is 0.3715 which means a tolerance of approximately $37.15 \%$ of coprime factor uncertainty.

\section{Simulation Results}

To demonstrate numerical results, we simulate the Tri-rotor UAV along with the designed controller in Simulink. Figure 5 depicts the block digram for the simulation where $\left[\begin{array}{ll}\eta_{r} & \lambda_{r}\end{array}\right]^{T}$ is the desired reference attitude and position respectively.

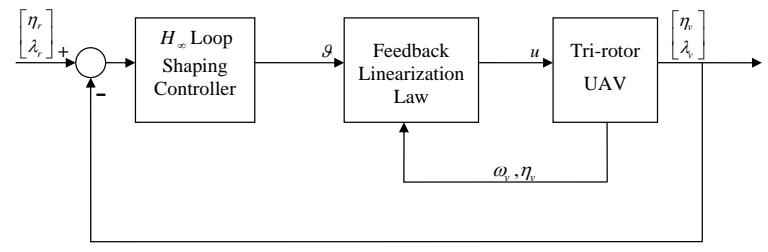

Figure 5. Simulation block diagram for the control design of the Tri-rotor UAV.

Figure 6 shows the singular values of the linearized plant, the shaped plant and the synthesized controller.

Figure 7 depicts the performance of the UAV for a scenario of horizontal hovering at $5 \mathrm{~m}$ height. The vehicle was at nonzero initial position and initial attitude as shown. The speed of the BLDC motors and the angles of the servo motors to stabilize the vehicle and track the reference signals are shown in Figure 8. The controller shows good performance with tracking in all channels. The servos and BLDC motors are not saturated and operate within their physical limits of $\pm 90^{\circ}$ for the servos and $11000 \mathrm{rpm}$ for the BLDC motors. 


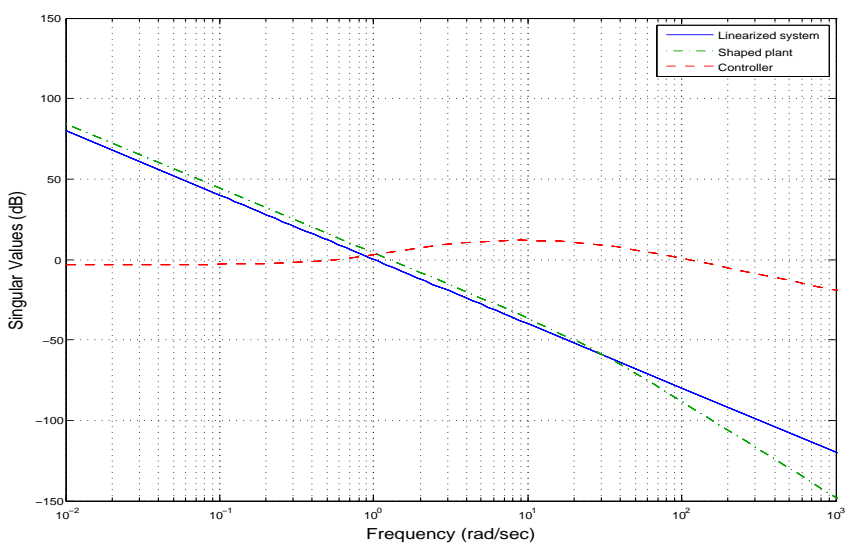

Figure 6. Singular value plots for the linearized system, the shaped system and the controller.
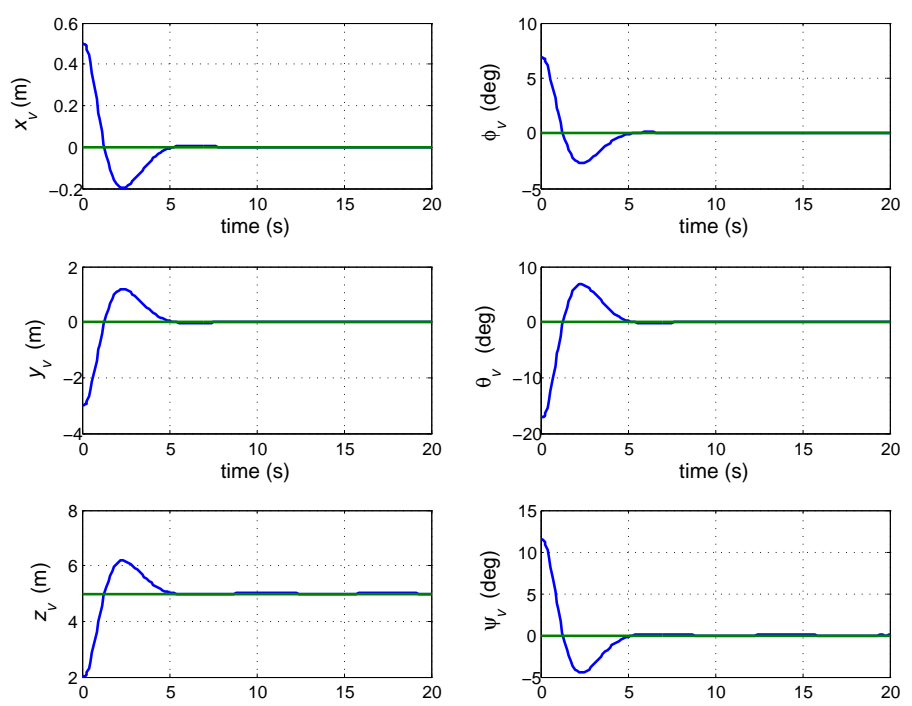

Figure 7. Simulation plots of the UAV position and attitude using the synthesized controller of $H_{\infty}$ loop shaping control associated with classical feedback linearization.
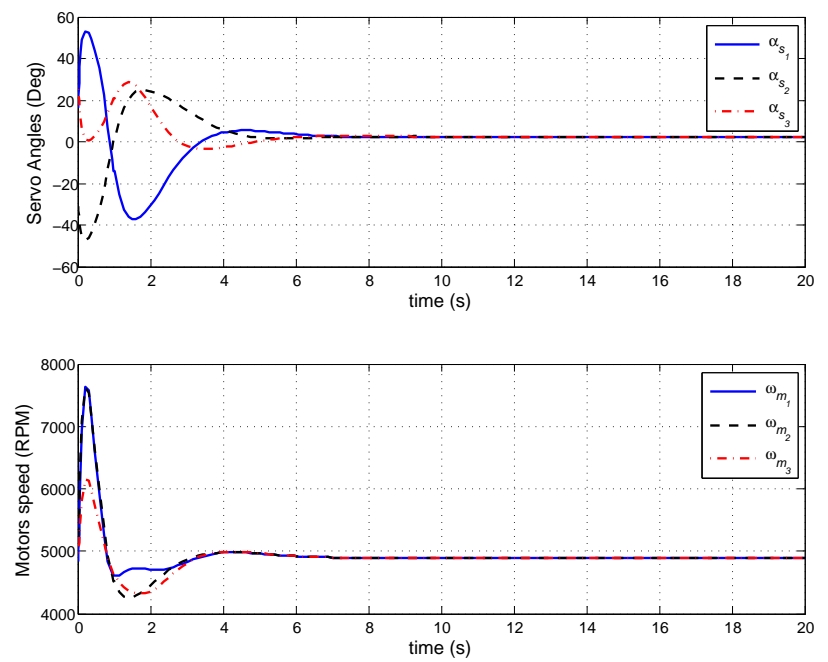

Figure 8. The performance of the actuators (servos and BLDC motors) to track the specified reference input of $(0,0,0)$ deg for attitude, and $(0,0,5) \mathrm{m}$ for position coming from non-zero initial point.

\section{CONCLUSION}

In this paper, a novel tri-rotor UAV is proposed. The proposed UAV has six actuators with full authority of thrust and torque vectoring. The mathematical model of the proposed design is non-linear and it indicates coupling between translational and rotational motion. The nonlinear model of the UAV is linearized by input-output feedback linearization. This procedure cancels the nonlinearity of all channels simultaneously without further conditions for specific operating point which is the case when we handle channels individually. The linearized plant is a double integrator that is controlled using $\mathscr{H}_{\infty}$ loop-design procedure. The result is verified via simulations. More complex feedback linearization techniques (such as robust feedback linearization in [15]) can be used in the same manner to avoid linearizing the system to a double integrator.

\section{REFERENCES}

[1] A. Das, K. Subbarao, and F. lewis, "Dynamic inversion with zerodynamics stabilisation for quadrotor contrl," IET Control Theory and Applications, vol. 3, pp. 303-314, 2009.

[2] D. J. Pines and F. Bohorquez, "Challenges facing future micro-airvehicle development," Journal of Aircraft, vol. 43, p. 290-305, 2006.

[3] K. P. Valavanis, Ed., Advances in Unmanned Aerial Vehicles: State of the Art and the Road to Autonomy, ser. International Series on Intelligent Systems, Control, and Automation: Science and Engineering. Springer, 2007, vol. 33.

[4] J. Escareno, A. Sanchez, O. Garcia, and R. Lozano, "Triple tilting rotor mini-UAV: Modeling and embedded control of the attitude," in American Control Conference, 2008, pp. 3476-3481.

[5] S. Salazar-Cruz, R. Lozano, and J. Escareño, "Stabilization and nonlinear control for a novel trirotor mini-aircraft," Control Engineering Practice, vol. 17, no. 8, pp. 886-894, August 2009.

[6] R. Huang, Y. Liu, and J. J. Zhu, "Guidance, navigation, and control system design for tripropeller vertical-takeoff-and-landing unmanned air vehicle," Journal of Aircraft, vol. 46, no. 6, pp. 1837-1856, NovemberDecember 2009.

[7] D.-W. Yoo, H.-D. Oh, D.-Y. Won, and M.-J. Tahk, "Dynamic modeling and stabilization technique for tri-rotor unmanned aerial vehicles." International Journal of Aeronautical and Space Science., vol. 11, pp. 167-174, 2010.

[8] P. Vanblyenburgh, "UAVs: An Overview," Air \& Space Europe, vol. 1, no. 5-6, pp. 43-47, September 1999.

[9] B. Crowther, A. Lanzon, M. Maya-Gonzalez, and D. Langkamp, "Kinematic analysis and control design for a non planar multirotor vehicle," Journal of Guidance, Control, and Dynamics, vol. 34, no. 4, pp. 11571171, 2011.

[10] F. Lin, W. Zhang, and R. D. Brandt, "Robust hovering control of a PVTOL aircraft," Control Systems Technology, IEEE Transactions on, vol. 7, no. 3, pp. 343-351, August 2002.

[11] P. Fan, X. Wang, and K.-Y. Cai, "Design and control of a tri-rotor aircraft," in Control and Automation (ICCA), 2010 8th IEEE International Conference on, June 2010, pp. 1972 -1977.

[12] Z. Prime, J. Sherwood, M. Smith, and A. Stabile, "Remote control (rc) vertical take-off and landing (vtol) model aircraft," LevelIV Honours Project Final Report, University of Adelaide, Adelaide, Australia, October 2005.

[13] G. D. Padfield, Helicopter Flight Dynamics: The Theory and Application of Flying Qualities and Simulation Modeling, ser. Education Series. AIAA, 1996.

[14] A. Lanzon, "Weight optimisation in $H_{\infty}$ loop-shaping," Automatica, vol. 41, no. 7, pp. 1201-1208, 2005.

[15] A. L. D. Franco, H. Bourlès, E. R. De-Pieri, and H. Guillard, "Robust nonlinear control associating robust feedback linearization and $H_{\infty}$ control," IEEE Transactions on Automatic Control, vol. 51, no. 7, pp. 1200-1206, 2006. 